\title{
An atypical case of optic disk drusen with nerve fiber layer thickening
}

This article was published in the following Dove Press journal:

Eye and Brain

26 May 2010

Number of times this article has been viewed

\author{
Burak Turgut \\ Mehmet Kaan Kaya \\ Tamer Demir \\ Firat University School of Medicine, \\ Department of Ophthalmology, \\ Elazig, Turkey
}

Correspondence: Burak Turgut Department of Ophthalmology Firat University School of Medicine, Elazig, Turkey

Tel +904242333555

Fax +90424238 8096

Email drburakturgut@gmail.com

\begin{abstract}
We report an atypical case of optic disk drusen (ODD). A 12-year-old girl complained of blurred vision in both eyes. However, visual acuities in both eyes were 20/20. Fundus examination revealed blurring of the optic disk margins and elevation of the optic disk in both eyes. Initially it was considered that the diagnosis would be papilledema. However, neurologic examination revealed no pathology which would cause the visual field defect and disk elevation. Optical coherence tomography (OCT) scans showed hyperreflectance lesions on the disk and shadowing areas beneath the optic nerve head in both eyes. Orbital ultrasound and orbital and cranial tomography revealed protuberances with hyperechogenicity and calcification in both eyes. It was considered that these findings were consistent with ODD. Repeated visual field tests showed constricted peripheral field loss in both eyes. Using OCT software, peripapillary nerve fiber layer thickening in both eyes corresponded to visual field defects in the superior and inferior quadrants, in which drusen was detected. Despite the extensive literature, in cases of ODD with blurred margins, elevated disks, and visual field defects, the peripapillary nerve fiber layer may be increased due to nerve layer infarct in the area which has calcification.
\end{abstract}

Keywords: optic disk drusen, pseudopapilledema, retinal nerve fiber, infarct, thickening

\section{Introduction}

Optic disk drusen (ODD) refers to a laminated calcified hyaline body located in the prelaminar portion of the optic nerve head. It results from calcific degeneration of axons within the optic nerve. ${ }^{1}$ There are two types of ODD. A visible drusen protrudes from the disk and is particularly prominent at the disk margin and the nasal portion of the optic disk, whereas buried ODDs are not directly visible, can cause elevation of the optic disk with blurred optic disk margins, and may be confused with disk edema. Thus, the diagnosis of buried ODD is clinically important. Visual manifestations of this entity include visual field defects and visual acuity loss. ${ }^{1-3}$ Localized nerve fiber layer thinning has been found to correspond with visual field defects in the quadrants in which drusen is detected. ${ }^{4}$ In this paper we report a case of ODD with nerve fiber layer thickening in quadrants where drusen were present.

\section{Case report}

A 12-year-old girl presented to our hospital complaining of blurred vision in both eyes. However, her corrected visual acuity was 20/20 in both eyes. She had no history remarkable for systemic or ocular disease. Her family ocular history was also unremarkable. The intraocular pressure was $17 \mathrm{mmHg}$ in both eyes. Direct and 


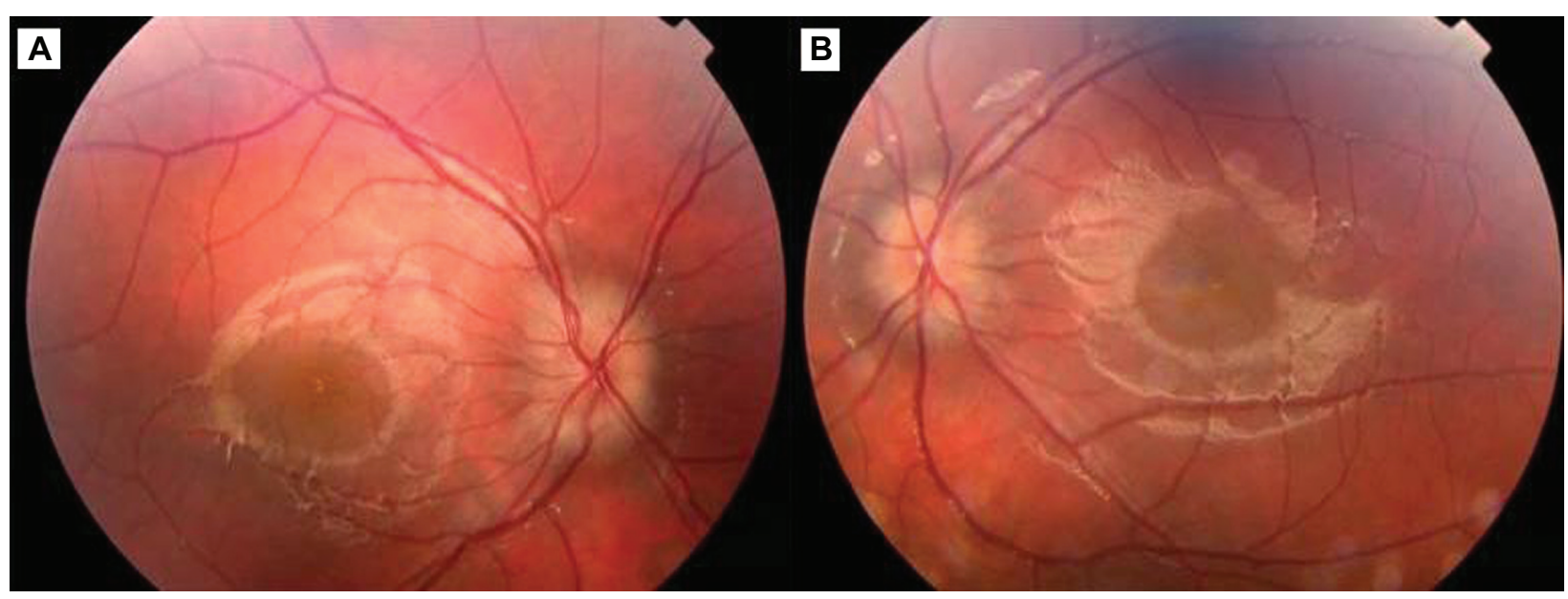

Figure I Blurring of optic disk margins and elevation of the optic nerve head in the right eye (A) and in the left eye (B).
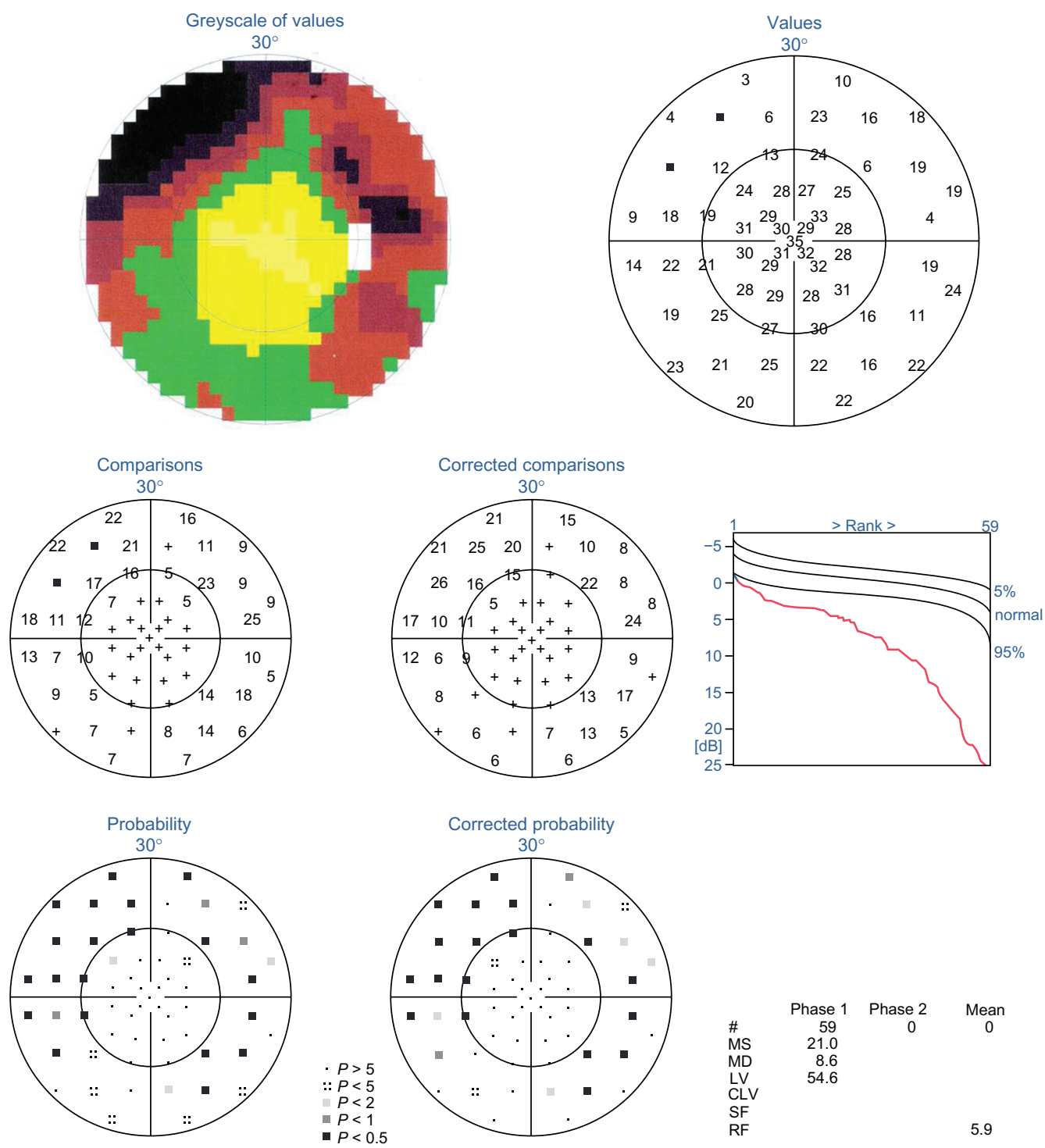

Figure 2 Peripherally concentric narrowing in the right eye.

Abbreviations: MS, mean sensitivity; MD, mean defect; LV, loss variance; CLV, corrected loss variance; SF, short-term fluctuation; RF, reliability factor. 
indirect light reflexes were intact, with no relative afferent pupillary defect. Extraocular movements were full. Anterior segment examination was normal, without iris pathology or cellular reaction. Fundus examination revealed blurring of the optic disk margins and elevation of the optic disk in both eyes (Figures 1A and 1B). Initially it was considered that the diagnosis would be papilledema. However, neurologic examination revealed no pathology which would cause visual field defects and disk elevation. Computed brain tomography showed no evidence of an acute infarct, or pituitary, suprasellar, or intracranial mass. Although orbital scans showed no evidence of abnormal enhancement or mass within orbit, a hyperechogenic elevated lesion in the optic nerve head was observed in both eyes. Fast optic disk scans using optical coherence tomography (OCT) performed by spectral OCT and a scanning laser ophthalmoscope showed hyperreflectance lesions on the disk and shadowing areas beneath the optic nerve head consistent with ODD. Orbital ultrasound and tomography revealed protuberances with hyperechogenicity and calcification in both eyes. It was considered that these findings were consistent with ODD. Repeated visual field tests (by Octopus 101 perimeter; Haag Streit Interziag Inc., Switzerland) showed peripheral concentric narrowing in both eyes (Figures 2 and 3). Using OCT software, peripapillary nerve fiber layer thickening corresponded to visual field defects in the superior and inferior quadrants in which the drusen was detected in both eyes (Figures 4, 5A, and 5B) Based on the clinical appearance of the optic disks and the ancillary tests, a diagnosis of buried ODD was made. It was recommended that the patient be re-examined in a further six
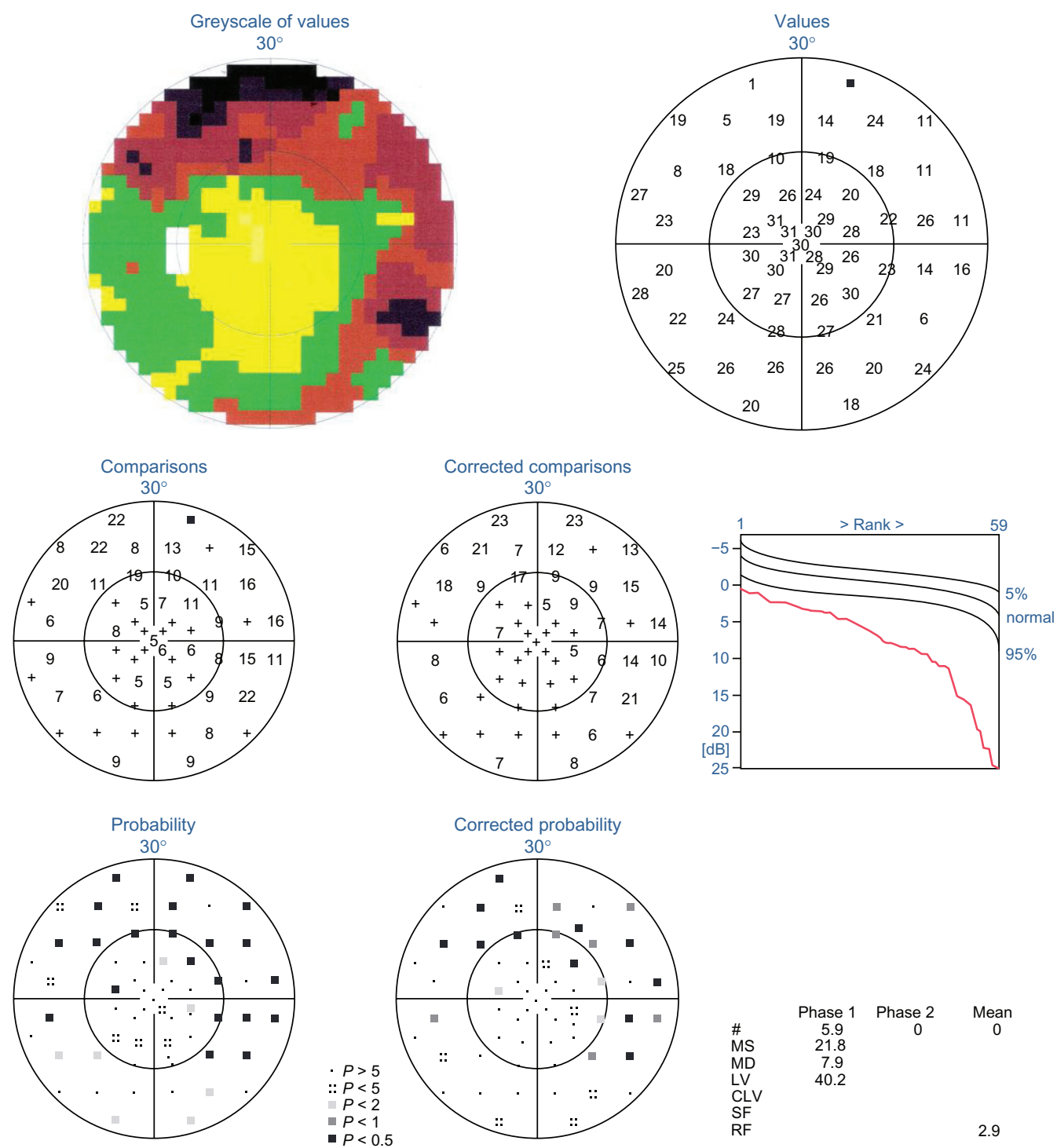

Figure 3 Peripherally concentric narrowing in the left eye.

Abbreviations: MS, mean sensitivity; MD, mean defect; LV, loss variance; CLV, corrected loss variance; SF, short-term fluctuation; RF, reliability factor. 

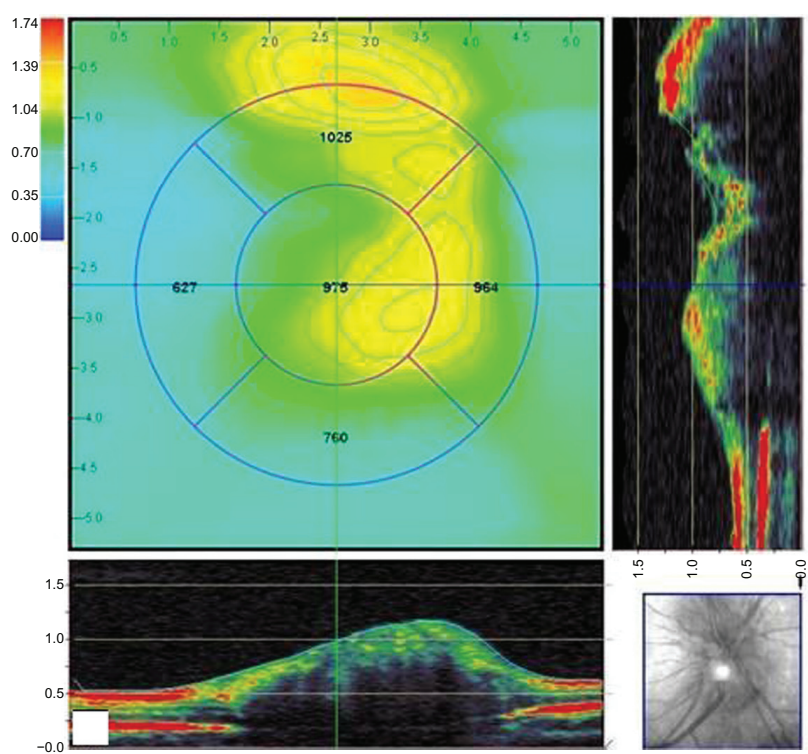
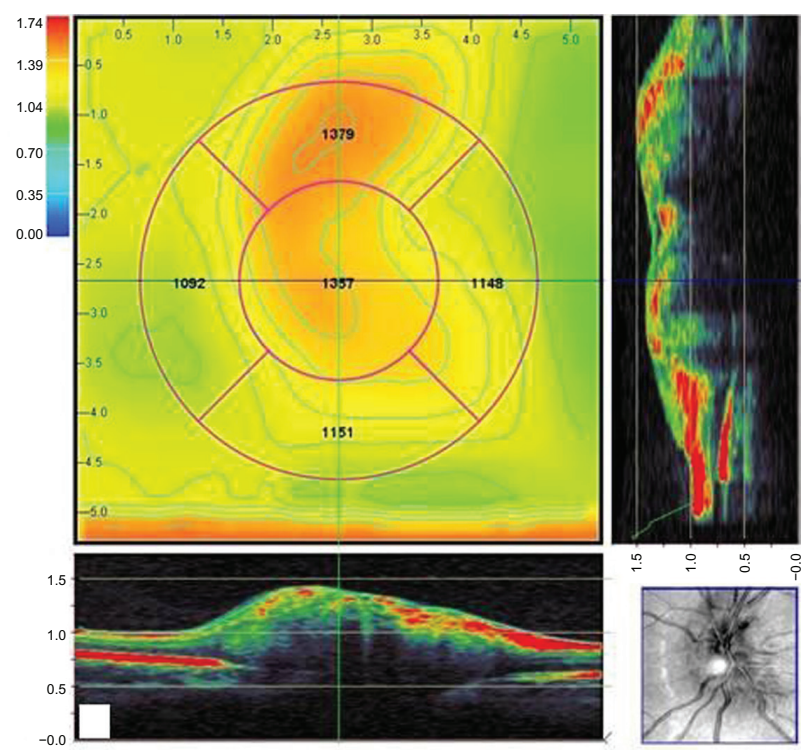

Figure 4 Optic disk tomography, vertical and horizontal scans provided by optical coherence tomography in the right eye (A) and the left eye (B).

months' time with ophthalmoscopy, automated visual fields, and retinal nerve fiber analysis. However, the patient has not returned for follow-up.

\section{Discussion}

The reported incidence of ODD is 3.4-4.9 per 1000 individuals, although a higher incidence of 20.4 per 1000 individuals has been reported in autopsy studies. ODD is bilateral in $75 \%$ of patients. Although the exact mechanism
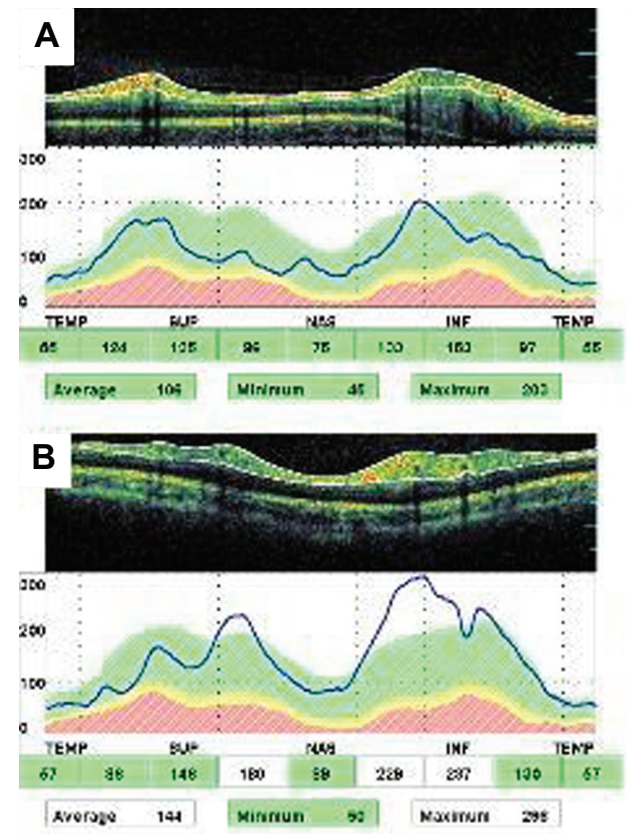

Figure $\mathbf{5}$ Thickening in the retinal nerve fiber layer in the right $(\mathbf{A})$ and the left eye (B). is unknown, factors which play a role in the pathogenesis of ODD are a small scleral canal, a congenital dysplastic disk, abnormal optic nerve vasculature, and degenerative axoplasmic transport. ${ }^{4-7}$ In cases of ODD, there may be the appearance of an elevated optic disk resembling papilledema. Although many patients are asymptomatic, there are reports of visual field and acuity loss. In recent studies, it was found that visible drusen produced more frequent and prominent visual field defects. ${ }^{4-7}$

ODD found in children are typically buried. Patients are typically asymptomatic or have mild visual field defects. Visual field defects reported in eyes with ODD include nerve fiber bundle defects, arcuate defects, enlargement of the blind spot, and concentric narrowing. Visual field defects are found to be more prevalent in eyes with superficial or visible drusen, and can appear during childhood, even preceding the clinical appearance of ODD. ${ }^{1-4,7}$

OCT in patients with ODD may show elevation of the optic disk and shadows on imaging. In recent studies which measured the nerve fiber layer using OCT in patients with ODD, it was found that localized nerve fiber layer thinning in quadrants corresponded to visual field defects on perimetry and in which the drusen were present. ${ }^{8-10}$ Retinal vascular events, including retinal artery occlusion, retinal peripapillary hemorrhage, and anterior ischemic optic neuropathy, have been reported in patients with ODD. ${ }^{1-4,7}$

Despite the extensive literature, in cases of ODD with blurred margins, elevated disks, and visual field defects, the peripapillary nerve fiber layer may be thickened due to nerve layer infarct and, subsequently, swelling of retinal 
nerve fibers in the area with calcification. However, the swollen appearance of the optic nerve or thickening of the nerve fiber layer might also be due to buried ODD.

\section{Disclosure}

The authors received no financial support for and have no proprietary interest in this research.

\section{References}

1. Davis PL, Jay WM. Optic nerve head drusen. Semin Ophthalmol. 2003;18:222-242.

2. Lam BL, Morais CG Jr, Pasol J. Drusen of the optic disc. Curr Neurol Neurosci Rep. 2008;8:404-408.

3. Gutteridge IF. Optic nerve drusen and pseudopapilledema. Am J Optom Physiol Opt. 1981;58:671-676.
4. Roh S, Noecker RJ, Schuman JS, et al. Effect of optic nerve head drusen on nerve fiber layer thickness. Ophthalmol. 1998;105:878-885.

5. Wilkins JM, Pomeranz HD. Visual manifestations of visible and buried optic disc drusen. J Neuroophthalmol. 2004;24:125-129.

6. Johnson LN, Diehl ML, Hamm CW, Sommerville DN, Petroski GF. Differentiating optic disc edema from optic nerve head drusen on optical coherence tomography. Arch Ophthalmol. 2009;127:45-49.

7. Yi K, Mujat M, Sun W, et al. Imaging of optic nerve head drusen: Improvements with spectral domain optical coherence tomography. J Glaucoma. 2009;18:373-378.

8. Morris RW, Ellerbrock JM, Hamp AM, et al. Advanced visual field loss secondary to optic nerve head drusen: Case report and literature review. Optometry. 2009;80:83-100.

9. Schuman JS, Puliafito CA, Fujimoto JG. Optical Coherence Tomography of Ocular Diseases. 2nd ed. Thorofare, NJ:Slack;2004.

10. Mustonen E, Nieminen H. Optic disc drusen - photographic study. II. Retinal nerve fiber layer photography. Acta Ophthalmol (Copenh). 1982;60:859-872.

Eye and Brain

\section{Publish your work in this journal}

Eye and Brain is an international, peer-reviewed, open access journal focusing on clinical and experimental research in the field of neuro-ophthalmology. All aspects of patient care are addressed within the journal as well as basic research. Papers covering original research, basic science, clinical and epidemiological studies, reviews and evaluations, guidelines, expert opinion and commentary, case reports and extended reports are welcome. The manuscript management system is completely online and includes a very quick and fair peer-review system, which is all easy to use. Visit http://www.dovepress.com/ testimonials.php to read real quotes from published authors. 